\title{
Commissioning Results of Slow Extraction of Heavy Ions from the AGS Booster*
}

\author{
K.A.Brown ${ }^{\dagger}$, L. Ahrens, S. Bellavia, S. Binello, B. Brelsford, D. DuMont,W. Eng, C. Gardner, \\ D. Gassner, J.W. Glenn, L.Hammons,J. Hock, L. Hoff, E. Hutchinson, J.Jamilkowski, \\ N. Kling, Y. Kotlyar, A. Krishock, R. Lockey,M. Mapes, I. Marneris, G. Marr, \\ A. McNerney, A.Meyer,J. Morris, C. Naylor, S. Nemesure, D. Phillips, A.Rusek, \\ J. Ryan, T. Shrey, L. Snydstrup, N. Tsoupas, B. VanKuik, S. Zahariou-Cohen, K. Zeno, \\ C-AD Dept., BNL, Upton, NY
}

\section{Abstract}

Brookhaven's AGS Booster has been modified to deliver slow extracted beam to a new beam line, the NASA Space Radiation Laboratory (NSRL). This facility was constructed in collaboration with NASA for the purpose of performing radiation effect studies for the NASA space program. The design of the resonant extraction system has been described in [1]. A more detailed description, which includes predictions of the slow extracted beam time structure has been described in [2]. In this report we will present results of the system commissioning and performance.

\section{INTRODUCTION}

The AGS Booster has operated since 1991 as an injector of protons and heavy ions into the AGS. The nominal operating parameters are summarized in table 1. NSRL will employ heavy ion beams of many different ion species and at beam energies ranging from 0.04 to $3.07 \mathrm{GeV} /$ nucleon. Resonant extraction is required in order to deliver a continuous stream of particles. In this report we present results from the commissioning of slow extraction for this facility. The third integer resonant extraction process employs a single thin magnetic septum and a thick septum ejector magnet, with the resonance created by re-configuring 4 of the main horizontal chromaticity sextupoles. The expected extraction efficiency is about $85 \%$, based on the thin septum thickness and the predicted step size of the resonant beam at the septum.

The maximum kinetic energy of the extracted beams is limited by the maximum Booster rigidity of $17 \mathrm{Tm}$ and by the maximum NSRL transport line rigidity of $13 \mathrm{Tm}$. Ions are fully stripped at a stripping foil located at the entrance of the thick septum magnet. Intensities of a few $10^{8}$ down to $10^{5}$ ions per pulse or less will be available to experimenters through collimation at the entrance to the D6 septum magnet. Due to the limited range of the Booster quadrupoles at high fields, the design of the resonant extraction system requires moving the operating point in tune space through $\nu_{x}=4.5$ and extracting on the $13 / 3$ resonance.

\footnotetext{
* Work performed under Contract Number DE-AC02-98CH10886 with the auspices of the US Department of Energy.

† kbrown@bnl.gov
}

Table 1: AGS Booster Parameters

\begin{tabular}{l|c}
\hline Parameter & Value \\
\hline Circumference & $201.78(1 / 4$ AGS $) \mathrm{m}$ \\
Ave. Radius & $32.114 \mathrm{~m}$ \\
Magnetic Bend R & $13.8656 \mathrm{~m}$ \\
Lattice Type & Separated Function, FODO \\
No. Superperiods & 6 \\
No. of Cells & 24 \\
Betatron Tunes, X,Y & $4.82,4.83$ \\
Vacuum Chamber & $70 \times 152 \mathrm{~mm}$ Dipoles \\
& $152 \mathrm{~mm}($ circular $)$ Quads \\
Max. Rigidity & $17 \mathrm{Tm}$ \\
Injection Rigidity & $2.2 \mathrm{Tm}(200 \mathrm{MeV}$ protons $)$ \\
& $0.9 \mathrm{Tm}(1 \mathrm{MeV} / \mathrm{nuc}$ Au $(32+))$ \\
Acceleration Rate & $8.9 \mathrm{~T} / \mathrm{s} \mathrm{up} \mathrm{to} 7.5 \mathrm{Tm}(7.5 \mathrm{~Hz})$ \\
& $1 \mathrm{~T} / \mathrm{s}$ up to $17 \mathrm{Tm}(0.7 \mathrm{~Hz})$ \\
\hline
\end{tabular}

\section{COMMISSIONING RESULTS}

The first slow extracted beam from the Booster occurred in October 2002. At that time we demonstrated that we could manipulate the beam in tune space with little or no beam loss while passing through $\nu_{x}=4.5$ and that the extraction system worked as designed. After this initial commissioning period we took occasional short periods of time to do various radiation safety beam tests. Our next opportunity for extensive commissioning effort began again at the end March, using polarized protons. This was useful time, since for the proton beams we could observe beam losses on the Booster ion chamber loss monitors. In this period of commissioning we were able to re-establish slow extraction, get beam transported to the target area of the NSRL beam line, commission new instrumentation in the beam line, and begin work on spill control. In addition the beam line itself was commissioned with various studies of the beam optics being performed. These commissioning efforts are discussed in more detail in [3, 4]. 


\section{Booster Main Magnet and Acceleration Configu- ration}

The Booster Main Magnet power supply required significant modifications to allow operation for slow extraction. In addition to modifications in the controls, an active filter was added, to reduce ripple on the extraction flattop. A spill servo system was added including beam abort operations, to disable beam in the middle of the spill through an experimenter supplied interrupt. Details of these changes can be found in [4]. Figure 5 shows the effect of the active filter on the spill structure.

\section{Tune Manipulations}

At high field, the Booster quadrupole iron begins to saturate before the dipole iron of the main arc bending magnets. This weakens the strength of the quadrupole relative to the dipole and lowers the machine bare tune. Details on this effect are provided in [5]. For the NSRL project new power supplies were installed to allow the trim windings of the main Booster quadrupoles to operate up to $1100 \mathrm{amp}$. Previously they could only go up to $700 \mathrm{amp}$, which was not enough to provide a good operating range for slow extraction at the highest rigidities. Figure 1 shows the regions of tune space that are accessible within $\pm 1100 \mathrm{amp}$ in the tune trim windings at $14 \mathrm{Tm}$, the larger region in green, and $16 \mathrm{Tm}$, the smaller region. For multiturn injection of $F e^{20+}$ and $F e^{21+}$, we use linear coupling to maximize the injection efficiency [6]. The nominal uncoupled tunes for this setup are near the point $\nu_{x}=4.757$ and $\nu_{y}=4.777$, indicated by the green dot in Figure 1. To get to the proper working point for slow extraction we first shift $\nu_{x}$ down to 4.4 at low energy where we are able to correct the half integer resonance using the normal stop band correctors. The tunes move along the path indicated by the dashed line in the figure, first to the blue point, and then to the black point. Figure 2 shows the effect of these manipulations on the circulating beam current. As can be seen we are able to make these changes in tune space with little or no beam loss.

\section{Extraction Bumps Configurations}

For control over step size at the thin septum and beam losses on the thick septum, the equilibrium orbit can be deformed at the thin and thick septum locations using a set of five individually controlled power supplies connected to trim coils on selected Booster Main sector magnets. Figure 3 shows a MAD simulation of the equilibrium orbit (for non-resonant beam) with the locations of the selected bump magnets indicated by the red arrows. The thin septum position and skew can be controlled through a set of motor drives connected to each end of the magnet. The thick septum is not movable, so the beam position at that location must be controlled by the bumps and the amount of excitation in the thin septum. The legend in the figure 3 shows the amount of current in the magnets, required to get the desired bump, for a beam rigidity of about $9.3 \mathrm{Tm}$. Dur-

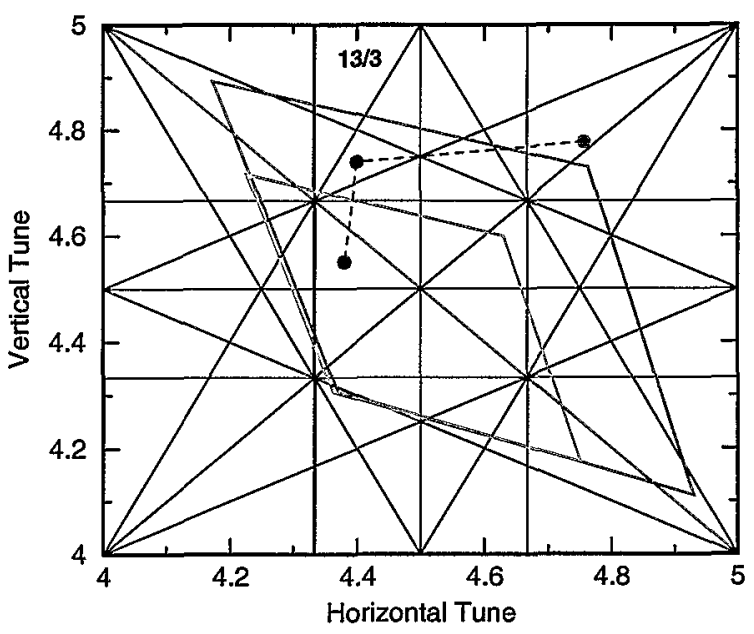

Figure 1: Booster Tune Space manipulations.

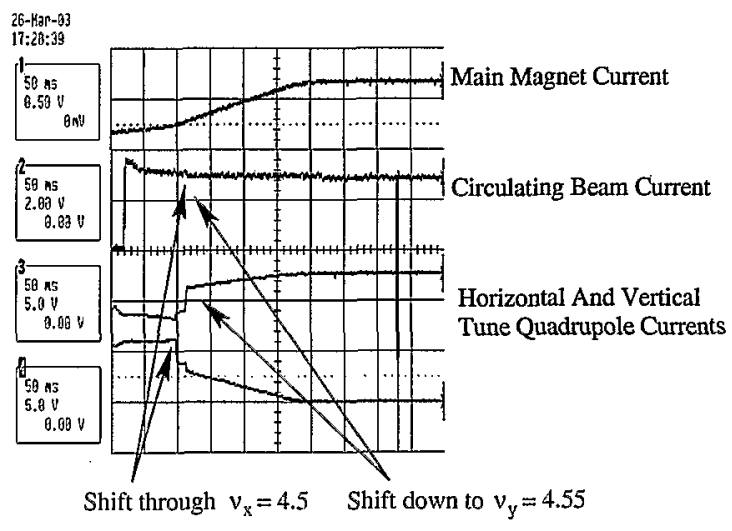

Figure 2: Beam Current Survival during tune manipulations.

ing commissioning we verified that the polarity of these power supplies was correct by exciting them individually and looking at single BPM signals.

\section{Resonance Excitation}

To finally extract the beam the resonant sextupoles have to be excited and the beam moved into the resonance by slowly lowering the field of the Booster Main Magnet. This causes the tune of the beam to be slowly shifted down into the resonance and particles enter into non-linear betatron amplitude growth. As the step size increases over many turns the particles eventually either hit the thin septum and are lost or step across it and are deflected across the thick septum and then bent out of the Booster into the NSRL beam line. Figure 4 shows the circulating beam current in the Booster slowly decreasing as beam enters the resonance and is extracted. Figure 5 shows the beam spill as seen on an external ion chamber in NSRL beam line. Finally figure 6 shows the beam as seen on a illumination flag in the beam line. 


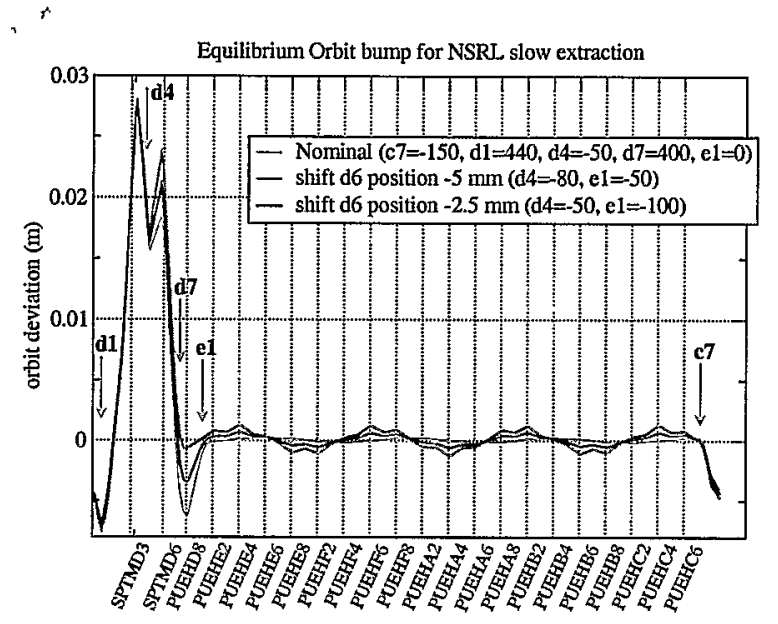

Figure 3: Equilibrium Orbit Deformation for Slow extraction.

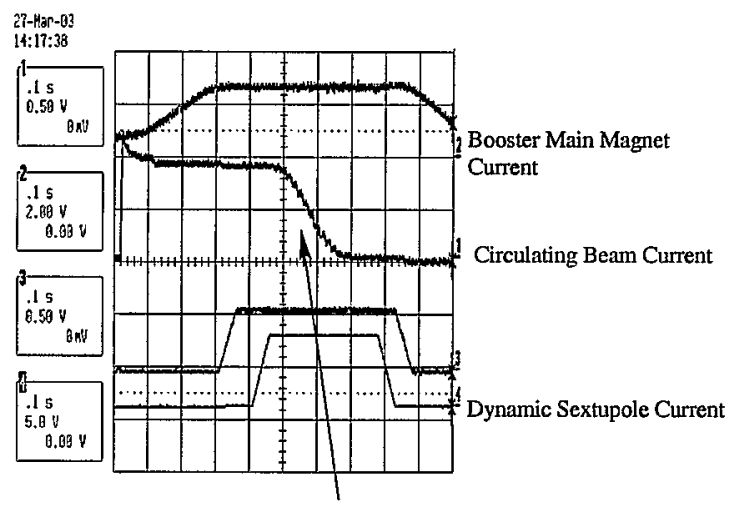

Beam Excited by Resonance and Extracting.

Figure 4: Beam Excited by Resonant Sextupoles and Extracting.

\section{REFERENCES}

[1] K.A.Brown, et al, "Design of a Resonant Extraction System for the AGS Booster", p. 1270, Proceedings of the 1999 Particle Accelerator Conference, New York, 1999.

[2] K.A.Brown, et al, "Resonant extraction parameters for the AGS Booster", p. 1571, Proceedings of the 2001 Particle Accelerator Conference, Chicago, 2001.

[3] N.Tsoupas, et al, "Commissioning of the Beam Transfer Line of the Booster Application Facility (BAF) at BNL", These Proceedings.

[4] I.Marneris, et al, "Booster Main Magnet Power Supply Improvements for NASA Space Radiation Laboratory at BNL", These Proceedings.

[5] K.A.Brown, et al, "A High Precision Model of AGS Booster Tune Control", Proceedings of the 2002 European Particle Accelerator Conference, Paris, 2002, p.548

[6] C.J.Gardner, et al, "Injection of Gold Ions in the AGS Booster with Linear Coupling", Proceedings of the 1999 Particle Accelerator Conference, New York, 1999, p.3294

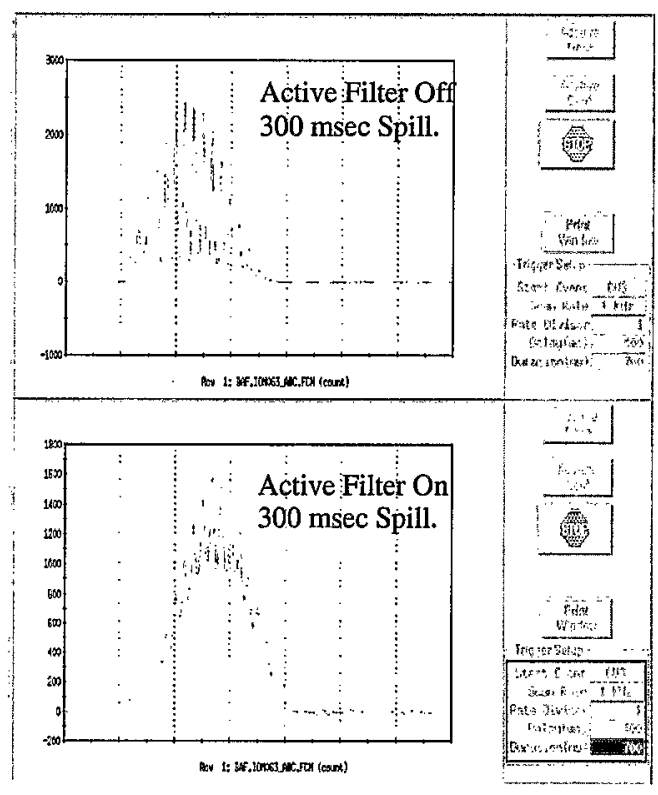

Figure 5: Beam Spill Seen on External Ion Chamber.

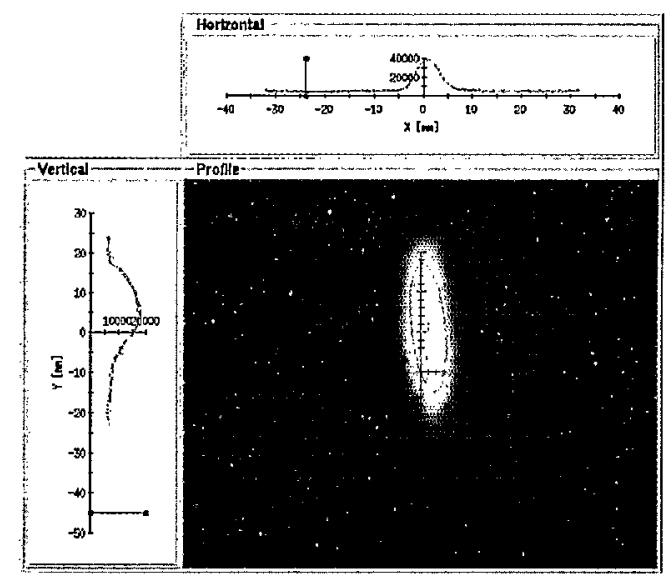

Figure 6: Beam As seen on external flag. 